Research paper

\title{
Icarus: In-situ monitoring of the surface degradation on a near-Sun asteroid
}

\author{
Tuomas Lehtinen $^{\mathrm{a}, \mathrm{b}, *}$, Mikael Granvik ${ }^{\mathrm{b}, \mathrm{a}}$, Andrea Bellome ${ }^{\mathrm{c}}$, Joan-Pau Sánchez ${ }^{\mathrm{c}}$ \\ ${ }^{a}$ Department of Physics, University of Helsinki, P.O. Box 64, 00014, Finland \\ ${ }^{\mathrm{b}}$ Asteroid Engineering Laboratory, Onboard Space Systems, Luleå, University of Technology, Box 848, S-98128 Kiruna, Sweden \\ c Space Research Group, Cranfield University, Cranfield, United Kingdom
}

\section{A R T I C L E I N F O}

\section{Keywords:}

Space mission

Asteroid

Rendezvous

Near-Sun

\begin{abstract}
A B S T R A C T
Icarus is a mission concept designed to record the activity of an asteroid during a close encounter with the Sun. The primary science goal of the mission is to unravel the nontrivial mechanism(s) that destroy asteroids on orbits with small perihelion distances. Understanding the destruction mechanism(s) allows us to constrain the bulk composition and interior structure of asteroids in general. The Icarus mission does not only aim to achieve its science goals but also functions as a technical demonstration of what a low-cost space mission can do. The proposed space segment will include a single spacecraft capable of surviving and operating in the harsh environment near the Sun. The spacecraft design relies on the heritage of missions such as Rosetta, MESSENGER, Parker Solar Probe, BepiColombo, and Solar Orbiter. The spacecraft will rendezvous with an asteroid during its perihelion passage and records the changes taking place on the asteroid's surface. The primary scientific payload has to be capable of imaging the asteroid's surface in high resolution using visual and near-infrared channels as well as collecting and analyzing particles that are ejected from the asteroid. The payload bay also allows for additional payloads relating to, for example, solar research. The Icarus spacecraft and the planned payloads have high technology readiness levels and the mission is aimed to fit the programmatic and cost constraints of the F1 mission (Comet Interceptor) by the European Space Agency. Considering the challenging nature of the Icarus trajectory and the fact that the next F-class mission opportunity (F2) is yet to be announced, we conclude that Icarus is feasible as an F-class mission when certain constraints such as a suitable launch configuration are met. A larger mission class, such as the M class by the European Space Agency, would be feasible in all circumstances.
\end{abstract}

\section{Introduction}

We present a mission concept that aims to identify the mechanisms that drive the observed activity on asteroids with small perihelion distances and eventually destroy them.

For more than two decades it was thought that most near-Earth asteroids are eventually destroyed in a collision with the Sun [1]. Recent models of the near-Earth-object (NEO) population have shown that there is a lack of known objects on orbits with small perihelion distances and that the explanation for the missing NEOs is nontrivial $[2,3]$. The only sensible explanation is that NEOs experience a supercatastrophic disruption when their perihelion distance $q$ becomes small enough but still significantly larger than that considered by Farinella et al. [1]. According to the recent NEO models, the disruption of decameter-scale and larger NEOs occur when 0.05 au $\lesssim q \lesssim 0.2$ au. Such disruption distances are nontrivial to explain because the simplest explanations such as tidal forces caused by the Sun and direct evaporation can immediately be ruled out $[2,3]$.
The interior characteristics of asteroids such as their bulk (material) composition and structure are major knowledge gaps in contemporary planetary science. The discovery of mainbelt comets [4] and the direct detection of water on asteroid (1) Ceres [5] suggest that our knowledge of the bulk composition of asteroids is biased, because it is mainly based on (remote) spectroscopic observations of asteroid surfaces and laboratory analysis of meteorites that survive atmospheric entry. If the mechanism causing the destruction of asteroids at small $q$ would be known, it would allow us to more accurately predict the distribution of meteoroids in the interplanetary space and pave the way to a deeper insight about the bulk composition of NEOs. Unfortunately, there is currently very limited knowledge of what exactly causes the destruction of NEOs at small perihelion distance irrespective of their material composition.

Asteroid (3200) Phaethon is currently the best-known example of an non-cometary body that is apparently undergoing thermally-driven surface degradation during a few days around its perihelion passage

\footnotetext{
* Corresponding author at: Department of Physics, University of Helsinki, P.O. Box 64, 00014, Finland.

E-mail addresses: tule@iki.fi (T. Lehtinen), mgranvik@iki.fi (M. Granvik).
} 
[6]. Comet 322P is another object that shows comet-like activity close to the Sun in SOHO data while no signs of activity can be observed at a heliocentric distance of 1-2 au suggesting that it may in fact be an asteroid [7]. We are aware of observation programs that have recently been initiated to look for comet-like activity on asteroids with small $q$. These programs will likely increase the sample of potentially interesting mission targets.

The surface degradation on Phaethon produces meteoroids that contribute to replenishing the Geminid meteoroid stream but at the current rate is not sufficient to sustain the Geminid meteor flux. Based on the relation between the absolute magnitude $(H)$ and the typical disruption distance $\left(q_{\star}\right)$ presented by Granvik et al. [2], Phaethon is not expected to disrupt on its present orbit given the combination of its size and perihelion distance.

JAXA's DESTINY ${ }^{+}$mission, which will be launched in 2022 according to the current schedule, will do a fast flyby of Phaethon and, potentially, of asteroid (155140) 2005 UD, which may be a fragment of Phaethon [8,9]. DESTINY ${ }^{+}$will also fly through the Geminid meteoroid stream. The Phaethon flyby will occur at a heliocentric distance $r \sim$ 1 au and Phaethon has never shown any activity at that distance from the Sun. Hence the DESTINY ${ }^{+}$mission is unlikely to provide direct evidence about the mechanism which causes the observed activity during Phaethon's perihelion passage.

Here we present Icarus, a mission concept that is complementary to the DESTINY ${ }^{+}$mission. The main goal of Icarus is to observe changes that happen to an asteroid during its perihelion passage and gain insight about the processes that produce the observed activity on asteroids at small $q$ and eventually destroy them completely. Icarus carries a high-resolution imager that covers visual (Vis) and nearinfrared (NIR) wavelengths as well as equipment to analyze gas and dust ejected from the asteroid. The concept is specifically designed within programmatic and cost-cap conditions comparable to those of an F-class mission by the European Space Agency (ESA) [10]. The constraints are primarily met by relying heavily on heritage missions and readily available components off the shelf (COTS). Some proposed parts might need modification, but all the underlying technology aboard the Icarus spacecraft is fully flight-tested and qualified. On the other hand, the trajectory design is a particularly challenging aspect of the mission (see detailed discussion in Section 5.3.)

A short overview of the mission (see Section 5.2 for a more detailed description):

- After launch the Icarus spacecraft will perform orbital maneuvers to reduce the perihelion distance of its orbit to $q \sim 0.2 \mathrm{au}$.

- Icarus will then rendezvous with a suitable asteroid - chosen based on the launch date and trajectory - at a heliocentric distance $r \sim 0.7 \mathrm{au}$, follow it through the perihelion passage at $r \sim 0.2$ au, and continue until $r \sim 0.7$ au again.

- During the perihelion passage Icarus will fly close enough to the target asteroid to successfully commission its payload suite and gather all the required scientific data.

- Icarus will stay operational for a few months after the perihelion passage maintaining a periodic ground contact.

- After about 6 months of science operations Icarus will be decommissioned. The whole mission is estimated to last from 7 to 9 years. It should be noted, however, that the currently considered targets follow a clear trend of increased time of flight with decreased $\Delta \mathrm{V}$. This introduces an important trade-off, to be considered in the next mission phases, between launch mass and total mission duration.

Some specific questions that the successful Icarus mission will answer are:

(a) What are the primary mechanisms causing the destruction of asteroids at small perihelion distances? (b) How does the mass-loss rate change before, during, and after the perihelion passage?

(c) What is expelled by the asteroid?

(d) Is there evidence for volatile elements?

In the Sections that follow, we first present the scientific motivation and science requirements for the mission (Sections 2 and 3, respectively). We then describe the mission implementation and the environment in Section 5, the space segment in Section 6, and examples for the scientific payload in Section 4. Finally, we offer our conclusions in Section 7.

\section{Scientific motivation}

The overarching Science Goals of the Icarus mission are to

- understand the mechanism or mechanisms that drive the activity and destroys asteroids on orbits with small perihelion distances, and

- improve our understanding of the meteoroid environment in the inner solar system.

During the recent years, several different mechanisms have been proposed to explain the activity and/or destruction of NEOs on small$q$ orbits. The proposed destruction mechanisms include, but are not limited to, thermal cracking [11] combined with radiation pressure that will blow away the resulting particles [12], the anisotropic emission of thermal photons [13] or the scattering of sublimating gas molecules [14] that lead to an increased spin rate and eventually destruction, and the violent release of pressure that has accumulated below the surface layer [cf. 15]. Whereas the previous mechanisms are caused by the thermal environment close to the Sun, Wiegert et al. [16] propose that the destruction of both SOHO comets and asteroids at small $q$ is at least partly caused by meteoroid erosion, that is, the removal of material through impacts by high-speed meteoroids.

Testing the hypotheses put forward is challenging because there are no in-situ observations of an ongoing destruction process. Also relevant direct measurements are missing such as an estimate of the meteoroid population close to the Sun. There are currently only a few indirect empirical constraints that can be used to test the hypotheses put forward. The most fundamental empirical constraint is that smaller NEOs are destroyed farther from the Sun Granvik et al. [2]. Kilometerscale bodies typically reach a critical perihelion distance $q_{\star} \sim 0.05 \mathrm{au}$ before being destroyed whereas decameter-scale bodies are typically destroyed at $q_{\star} \sim 0.2$ au.

A linear extrapolation of the $\left(H, q_{\star}\right)$ relation suggest that bodies with diameters less than one meter and with perihelion distances similar to the semimajor axis of Mercury ( $a \sim 0.4 \mathrm{au}$ ) should have been destroyed. This is indeed what has been verified to be the case based on the orbits of observed meter-scale Earth impactors [17]. The upper limit for the sizes of the fragments produced in a sequence of disruptions is not well constrained at the moment, but millimeter-scales have been suggested based on meteor data [16].

While all NEOs that approach the Sun are eventually destroyed, darker NEOs tend to be destroyed farther from the Sun $[2,18]$. This makes sense if the destruction mechanism is sensitive to, e.g., the volatile content, because darker, carbonaceous NEOs are thought to contain more volatiles than their brighter, silicate-rich counterparts [19].

Graves et al. [20] showed that the spectral slopes of NEOs belonging to spectral types $\mathrm{S}$ and $\mathrm{Q}$ become less reddened with a smaller perihelion distance. This suggests that the same mechanism that destroys NEOs at very small $q$ may just be refreshing the surfaces at larger $q$.

The specific Science Objectives of the Icarus mission are hence to

- quantify the changes incurred by the perihelion passage to the target asteroid's shape and surface characteristics, 
Table 1

Summary of the basic science requirements.

\begin{tabular}{lll}
\hline Target & Conditions & Science \\
\hline $\begin{array}{l}\text { Asteroid with perihelion distance } q \lesssim 0.2 \mathrm{au} \\
\text { and aphelion distance } Q \gtrsim 0.7 \mathrm{au} .\end{array}$ & Rendezvous with target asteroid. & In-situ asteroid monitoring. \\
$\begin{array}{l}\text { Asteroid surface (high resolution). } \\
\text { Asteroid surface (large FOV). }\end{array}$ & $\begin{array}{l}\text { Suitable altitude for } \sim 10 \mathrm{~cm} / \mathrm{px} \\
\text { resolution (baseline }<5 \mathrm{~km} \text { ). }\end{array}$ & $\begin{array}{l}\text { Visual data of changes on asteroid } \\
\text { surface in centimeter resolution. }\end{array}$ \\
$\begin{array}{l}\text { Suitable altitudes for global imaging. } \\
\begin{array}{l}\text { Asteroid surface (large wavelength } \\
\text { coverage). }\end{array}\end{array}$ & $\begin{array}{l}\text { Images of the asteroid and visual data of } \\
\text { global changes on asteroid surface. }\end{array}$ \\
Near asteroid environment. & $\begin{array}{l}\text { Suitable altitude for } \sim 2.5 \mathrm{~cm} / \mathrm{px} \\
\text { resolution (baseline }<5 \mathrm{~km} \text { ). }\end{array}$ & $\begin{array}{l}\text { High range (up to 2500 nm) spectral } \\
\text { data of asteroid surface. }\end{array}$ \\
\hline
\end{tabular}

- determine the surface composition of the target asteroid,

- quantify the mass-loss rate throughout the perihelion passage,

- identify the source(s) of the activity on the target asteroid, and

- determine the composition and size distribution of the ejected material.

The target asteroid has to meet some scientific constraints to ensure that the Science Goals and Science Objectives are met. Given our current knowledge about the destruction of NEOs at small $q$, the destruction mechanism(s) is active on asteroids with $q \lesssim 0.2$ au. The destruction mechanism should also be active on objects with larger $q$, say, $q \lesssim 0.4$ au, but we do not have solid evidence for that from direct observations or debiased population models. Aiming for a target with $q \sim 0.2$ au should thus ensure that the mission is both feasible and scientifically successful. In general, aiming for a target with an even smaller $q$ would be preferable, because the destruction is most likely occurring at a higher rate at smaller $q$ and the measurements are therefore easier to make and interpret.

The destruction of NEOs at small $q$ is visible in the NEO $q$ distribution up to $q \sim 0.7$ au as a lack of detected NEOs at small $q$ compared to model predictions [2]. Based on dynamical modeling, the destruction process itself is not affecting the $q$ distribution at $q \sim 0.7$ au [2]. The discrepancy between the observed and predicted $q$ distribution at $q \sim 0.7$ au is instead a secondary effect caused by the typically non-monotonic evolution of $q$ [see, e.g., Fig. 8 in 21]. That is, some small fraction of the test asteroids that should, in reality, be destroyed at small $q$ would erroneously contribute to the theoretically-predicted distribution at larger $q$ when their $q$ increases, if the destruction is not accounted for in the models. For $q \gtrsim 0.7$ au there is no population-level evidence for the destruction of entire asteroids. Orbital models also show that most asteroids that reach a small $q$ will have an aphelion distance $Q \gg 0.7$ au [3]. When combined these pieces of information imply that the destruction process is typically periodic. For understanding the typical destruction process one should therefore focus on objects that undergo periodic destruction phases which implies that their $Q \gtrsim 0.7$ au. For the same reason the monitoring should start when the target is more or less inactive, that is, at a heliocentric distance $r \sim 0.7 \mathrm{au}$, and monitor it throughout the perihelion passage until it evolves back out to $r \sim 0.7$ au.

As for the target size there are currently no strong constraints, because meter-scale NEOs are apparently destroyed at $q \sim 0.4$ au [16] and boulders on the surfaces of larger objects should be similarly destroyed at the same distance. Hence all NEOs that reach a sufficiently small $q$ are destroyed, but the smaller ones run out of material earlier than the larger ones that thus have time to evolve to a smaller $q$. A practical scientific constraint is to ensure that the target asteroid chosen is large enough to survive the perihelion passage so that it can be monitored also on the outbound trajectory. An orbit with $q \sim 0.2$ au corresponds to the average destruction distance for NEOs with diameters of a few tens of meters. Hence a target with a diameter of at least $100-200 \mathrm{~m}$ is adequate.
Table 2

Summary of key characteristics of the preliminary payload suite.

\begin{tabular}{lll}
\hline Parameter & Value & Notes \\
\hline Narrow-angle resolution & $18.6 \mu \mathrm{rad} / \mathrm{px}$ & Rendezvous distance $<5 \mathrm{~km}$. \\
Wide-angle resolution & $101 \mu \mathrm{rad} / \mathrm{px}$ & FOV $11.35^{\circ} \times 12.11^{\circ}$ \\
Spectral range & $250-2500 \mathrm{~nm}$ & VIS-NIR imagers, SWIR detector \\
Total mass & $\sim 47 \mathrm{~kg}$ & With design margin. \\
Total power & $\sim 130 \mathrm{~W}$ & With design margin. \\
Total volume & $\sim 0.47 \mathrm{~m}^{3}$ & With design margin. \\
\hline
\end{tabular}

\section{Science requirements}

The Science Objectives described in Section 2 lead to the Science Requirements discussed below (for a summary, see Table 1).

The mission calls for in-situ monitoring of an asteroidal object with perihelion distance $q \lesssim 0.2$ au and aphelion distance $Q \gtrsim 0.7$ au prior, during, and after its perihelion passage.

Visual (Vis) high-resolution imaging of the asteroid's surface with centimeter-scale resolution is required to pin-point macroscopic changes in the surface constituents. A preliminary estimate for the required resolution is $<20 \mu \mathrm{rad} / \mathrm{px}$ which would correspond to $\sim 10 \mathrm{~cm} / \mathrm{px}$ from a distance of $5 \mathrm{~km}$. The high-resolution imager should cover a wavelength range of $250-1000 \mathrm{~nm}$. The field of view (FOV) of the instrument should also enable global monitoring of the asteroid.

Hyperspectral imaging of the asteroid surface will provide information about, for example, the composition of the asteroid. The hyperspectral imager does not need to have as high a resolution as the high-resolution imager, but should still provide $<600 \mu \mathrm{rad} / \mathrm{px}$ corresponding to $\sim 2.5 \mathrm{~m} / \mathrm{px}$ from a distance of $5 \mathrm{~km}$. The instrument should be capable of Vis to near-IR imaging up to $2500 \mathrm{~nm}$.

The science objectives also require collection and analysis of the material expelled from the asteroid. If the material expulsion is driven by thermal changes then this activity will increase during and immediately after the perihelion passage. The instrument for particle collection and analysis is required to detect both refractory and volatile elements. In addition, measurements of the meteoroid environment in the vicinity of the asteroid would be useful.

\section{Outline of the science payload}

\subsection{Example payload suite}

The mission requires at least three different instruments, complying with the Science Requirements, to fulfill its Science Objectives and, ultimately, Science Goals. The options listed in the following sections overlay an example payload suite, which functions as a proof of concept that suitable instrument architecture already exists and is at least partly space qualified. A summary of the key characteristics are provided in Table 2. 


\subsubsection{High-resolution imager}

We chose the Optical, Spectroscopic, and Infrared Remote Imaging System (OSIRIS), flown with the Rosetta spacecraft, for the preliminary analysis [22]. OSIRIS has an approximate size of $800 \times 1000 \times 500 \mathrm{~mm}^{3}$ with a total weight of about $35 \mathrm{~kg}$ including a high-resolution Narrow Angle Camera (NAC), a Wide Angle Camera (WAC), and electronics boxes. The NAC images over a wavelength range of $250-1000 \mathrm{~nm}$ with an angular resolution of $18.6 \mu \mathrm{rad} / \mathrm{px}$. The WAC has a wider FOV with a resolution of $101 \mu \mathrm{rad} / \mathrm{px}$. By imaging a 1-km-diameter asteroid from a distance of around $5 \mathrm{~km}$ the NAC (with a FOV of $2.20^{\circ} \times 2.22^{\circ}$ ) can resolve around $9 \mathrm{~cm}$ per pixel. From the same distance the asteroid fills the entire FOV of WAC $\left(11.35^{\circ} \times 12.11^{\circ}\right)$. The OSIRIS camera system is flight proven and all the underlying technology is available.

\subsubsection{Hyperspectral imager}

As the baseline for the hyperspectral imager, we chose the Asteroid Spectral Imager (ASPECT), which is currently in development for the Milani (previously APEX) CubeSat [23] to be launched as a part of ESA's Hera mission. The imager utilizes visual (VIS), near-infrared (NIR) and short-wavelength infrared (SWIR) channels to gain a wavelength range of $500-2500 \mathrm{~nm}$. The size and power requirements are moderate $\left(97 \times 97 \times 100 \mathrm{~mm}^{3}\right.$ and $10 \mathrm{~W}$, respectively). The spectral capability is achieved by a tunable Fabry-Perot Inferometer. The instrument currently has a Technology Readiness Level (TRL) of 5/6, which is acceptable for an ESA F-class mission. The underlying architecture of the instrument is, however, based on space-qualified designs of the instruments flown on Aalto-1 and Picasso VISION [24,25].

\subsubsection{Particle collection and analysis}

A particle detector and/or mass spectrometer is needed to study the material expelled from the asteroid. The Compact Ion and Neutral Mass Spectrometer (INMS), developed for the Dellingr CubeSat that was launched in 2017, is a good candidate [26]. The instrument offers in-situ measurements of ionized and neutral $\mathrm{H}, \mathrm{He}, \mathrm{N}, \mathrm{O}, \mathrm{N}_{2}$, and $\mathrm{O}_{2}$ densities. The instrument is compact $\left(110 \times 100 \times 100 \mathrm{~mm}^{3}\right)$, relatively lightweight $(600 \mathrm{~g})$, does not require a lot of power $(1.2 \mathrm{~W})$, and the design is flight proven.

Inclusion of a dust detector is desirable since it helps the navigation system to stay out of the asteroid tail while simultaneously providing scientific data about the near-asteroid environment. A Piezo Dust Detector (PDD), designed for the Armadillo mission that was launched in June 2019, is specifically developed to be a cost-effective solution [27]. One detector includes 9 elements with a total size of $80 \times 80 \times 40 \mathrm{~mm}^{3}$, weighs less than $500 \mathrm{~g}$ and uses a small amount of power $(3 \mathrm{~W})$. It can detect particles with velocities up to $10 \mathrm{~km} / \mathrm{s}$ from $1 \mu \mathrm{m}$ to $1 \mathrm{~mm}$.

\subsection{Additional instrumentation}

Depending on the final launch capabilities, additional modular instrumentation could be added to the Icarus payload suite. Approximate constraints on the additional instrumentation are the following:

- The size should be around $300 \times 100 \times 100 \mathrm{~mm}^{3}$ (3U).

- The maximum mass is $\sim 4 \mathrm{~kg}$.

- The TRL of the proposed instrument has to be at least 5/6.

We note that these constraints may change because they depend on the final configuration of the spacecraft.

\section{Mission implementation}

As per ESA's F-class mission constraints and requirements (Section 5.1), the design of the mission has to be conservative and innovative while still providing reliable hardware solutions. Furthermore, the mission has to be designed to be in agreement with the standards defined by the European Cooperation for Space Standardization (ECSS). The Icarus mission shall achieve its primary scientific goal, be ready to launch within 10 years from the next F-class mission call, and adapt mass and power margins of $20 \%$ as defined by ECSS for concept missions.
Table 3

An indicative cost breakdown provided by ESA [10].

\begin{tabular}{lll}
\hline Item & Cost & Notes \\
\hline Space segment & $\sim 65 \%$ & Includes all elements to be funded by ESA. \\
ESA project & $\sim 15 \%$ & $\sim 20 \%$ of the cost of the space segment. \\
Spacecraft operations & $\sim 20 \%$ & Strongly depends on the mission profile. \\
Launch costs & $0 \%$ & Covered by ESA. \\
\hline
\end{tabular}

\subsection{Constraints and requirements}

For the programmatic constraints we use those that were announced for the F1 mission call in 2018 [10], because no new F-class calls are open at the moment. We thus assume that the main constraints for a future F-class mission are that

- the maximum wet mass has to be less than $1000 \mathrm{~kg}$,

- the Cost at Completion (CaC) has to be less than 150M euros (not including launch costs; Table 3),

- the TRL of the spacecraft platform needs to be at least 7 by mission adoption,

- the total mass of the instrument(s) should not exceed $80 \mathrm{~kg}$,

- the TRL of the instrument(s) needs to be at least $5 / 6$ by mission adoption,

- the nominal science operations have to last less than 2 years not including the cruise phase.

The most relevant design constraints relate to the mission environment and mission profile:

i. the spacecraft shall carry out science operations at heliocentric distance $0.2 \mathrm{au} \lesssim r \lesssim 0.7 \mathrm{au}$,

ii. the spacecraft shall rendezvous with an asteroid at a distance less than $5 \mathrm{~km}$,

iii. the spacecraft shall withstand the solar irradiance $(\sim 35000$ $\mathrm{W} / \mathrm{m}^{2}$ ) during closest approach to the Sun,

iv. the spacecraft shall provide sufficient power at a heliocentric distance $0.2 \mathrm{au} \lesssim r \lesssim 1.5 \mathrm{au}$,

v. the spacecraft shall accommodate for a high-capacity internal memory $(\sim 380 \mathrm{~Gb})$,

vi. all the recorded data shall be returned without significant losses,

vii. space shall be allocated for primary science payloads as well as potential additional payloads,

viii. the spacecraft shall be compatible with all in-orbit environments (Section 5.4), for example via radiation hardening or thermal rejection,

ix. the communications subsystem shall communicate, at adequate rates and bands (uplink/downlink), with ground stations,

$\mathrm{x}$. the on-board data handling subsystem shall be able to process and store all the scientific and housekeeping data produced,

xi. the power subsystem shall keep all vital subsystems running at all times,

xii. the propulsion subsystem shall complete all the necessary orbital maneuvers in order to complete the mission,

xiii. the attitude and orbit control subsystem (AOCS) as well as the guidance, navigation and control (GNC) system shall be able to navigate and determine spacecraft position and attitude and provide corrections to both.

\subsection{Overview of mission operations}

The Icarus mission can be divided into relevant operational phases. These include: launch and early operations phase (LEOP), in-orbit commissioning phase (IOCP), operational phase (OP), and end-of-life (EOL) phase. We will next discuss the main events of each phase.

Before and during launch the spacecraft will be in stand-by-mode (SBM) and only the necessary subsystems (i.e. communications) are 


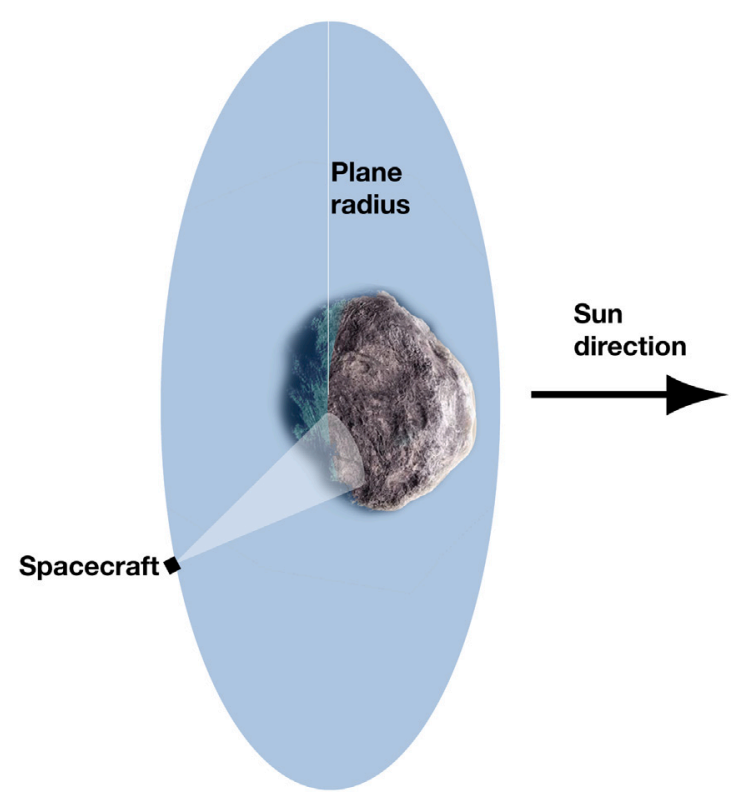

Fig. 1. The spacecraft images the terminator of the asteroid.

on, powered by the battery system on-board. After separation from the main stage in LEOP an initial acquisition mode (IAM) is entered. In IAM the spacecraft will find nominal attitude and deploy the solar arrays and communications hardware. The IAM is completed when the spacecraft receives solar power and establishes contact with the Earth. Once Icarus is in its operational configuration, the IOCP begins during which operation and system performance is validated and the early operational orbit is established. This phase can take up to a few weeks.

When all the systems have been validated and the IOCP is complete, the spacecraft enters its OP where it will remain for the majority of the mission duration. The OP includes all the mission related tasks such as orbit acquisition and maneuvers, cruise phase and navigation, and scientific data collection (science operations). The cruise phase includes a transfer period which can last up to 8 years depending on the chosen mission profile (see Section 5.3.2). The spacecraft will work according to a schedule uploaded from ground, which can be updated and patched if and when needed.

Icarus will commence science operations once the spacecraft is in rendezvous with the target asteroid and at $r \sim 0.7$ au. The main task during science operations is fine pointing, meaning that the spacecraft will remain in such an orientation and distance that the payload suite (see Section 4) has time to gather the required amount of data. Icarus will also establish ground contact whenever possible throughout the mission. The planned mission profile has the spacecraft in a plane around the asteroid where the asteroid's terminator can be observed (Fig. 1). This is done so that the contrast between the sunlit and shadowed regions can be imaged simultaneously giving a diverse data set from a single viewpoint. For example, the thermal gradient of the surface is strong at the terminator, and it is thus a region that most likely shows significant activity. All of the orbital and attitude maneuvers conducted during the science operations are done conservatively and maintaining a safe attitude relative to the Sun. Any proposed additional mission objectives will be performed during the OP and all of the additional mission data will be treated similarly to the nominal data. The duration of the science operations is estimated at less than 6 months, with about 60 days for science operations followed by a maximum of 40 days of downlink time (see Section 6.1).

If something goes wrong at any stage of the mission, Icarus will enter a safe mode (SM). The goal is to remain in safe attitude (i.e., the heat shield pointed toward the Sun) while keeping the ground link available.
To ensure spacecraft safety, the next iterations of the Icarus concept has to consider communication blackouts due to solar conjunctions.

After the OP the mission will move to the EOL phase. If the final orbit does not put Icarus in the immediate vicinity of the Earth, no deorbit maneuvers are needed. However, if the final mission cost budget allows for it, or if additional sponsorship from ESA Member States or partners is secured, a possibility for a non-conventional EOL exists. Instead of disconnecting power supplies and emptying propellant tanks, the spacecraft would move into post-mission operations and be left in small- $q$ orbit transmitting simple housekeeping data (see estimate in Section 6) while performing a system-wide stress test in the extreme heat of the Sun. Eventually the Icarus spacecraft will fail and be destroyed, thereby living up to its name.

The total Icarus mission duration is estimated to last 7-9 years, which will mostly depend on the launch, final propulsion configuration (i.e., chemical or electric propulsion system) and $\Delta V$ budget (see further discussion in Sections 5.3.2 and 6). It is thus likely that the mission duration may be longer than that allowed by the ESA F-class mission constraints, which state that the maximum mission duration is 5.5 years including a 5 -year cruise phase followed by 6 months of science operations [10]. However, an early estimation of the Icarus space segment cost shows that some of the allocated budget could be transferred to the operations costs allowing for an additional 24 years of mission operations time if chemical propulsion is chosen or 1-1.5 years if choosing an electric propulsion system. Sponsorship from ESA Member States or partners could further ensure that the Icarus mission complies with the cost-cap set by ESA for F-class missions.

\subsection{Baseline mission design}

The design of the trajectory is built on requirements and constraints derived from two sources: the mission Science Objectives and ESA's boundary conditions from the first F-class mission call [10]. The following sections show the first steps toward a suitable baseline trajectory, that follow the aforementioned requirements and constraints.

\subsubsection{Target asteroid}

Potential target asteroids must, as discussed in Section 2, have a perihelion distance $q \lesssim 0.2$ au as well as a reasonable size (i.e., absolute magnitude $H \lesssim 22$ ). As of December 2020, 63 such asteroids are known. However, it should also be noted that, by the time Icarus is launched, many more such objects should have been discovered. The next available mission and launch opportunity for Icarus would be the next potential F-class mission (F2), which is expected to share the launch with ESA's M5 mission (either Theseus or EnVision) in 2032. Due to the unknown launch date and configuration, it is essential to have enough candidate targets to choose from to accommodate different scenarios.

In addition to asteroids discovered by the leading ongoing asteroid surveys such as Catalina Sky Survey (CSS), Panoramic Survey Telescope And Rapid Response System (Pan-STARRS), and Asteroid Terrestrialimpact Last Alert System (ATLAS), Icarus' list of candidate targets will include discoveries by the Vera C. Rubin Observatory Legacy Survey of Space and Time (LSST). LSST is scheduled to start in late 2023 and it will be the most extensive NEO survey ever performed [28]. Table 4 provides a set of example targets for Icarus with $q \sim 0.2$ au and orbits for some of them in Fig. 2.

\subsubsection{Preliminary trajectory design}

The velocity at infinity $v_{\infty}$ required to inject a spacecraft into a small- $q$ orbit directly from Earth goes beyond the capabilities of current launch vehicles [33]. It is thus necessary to design a multiplegravity assist (MGA) trajectory to change the spacecraft energy with respect to the Sun by exploiting close passages with celestial bodies. NASA's Parker Solar Probe [34] and ESA's Solar Orbiter [35] are recent examples of MGA missions for small- $q$ target orbits around the Sun. 
Table 4

Examples of candidate targets for the Icarus mission. $q$ is the perihelion distance, $Q$ is the aphelion distance, $P$ is the orbital period, and $D$ the effective diameter.

\begin{tabular}{lllrl}
\hline Designation & $q[\mathrm{au}]$ & $Q[\mathrm{au}]$ & $P[\mathrm{~d}]$ & $D[\mathrm{~km}]$ \\
\hline$(153201) 2000 \mathrm{WO}_{107}$ & 0.200 & 1.623 & 318 & 0.5 \\
$(387505) 1998 \mathrm{KN}_{3}$ & 0.195 & 2.888 & 699 & 1 \\
$(455426) 2003 \mathrm{MT}_{9}$ & 0.197 & 4.868 & 1472 & 0.7 \\
$(506491) 2003 \mathrm{UW}_{29}$ & 0.189 & 2.150 & 462 & 0.2 \\
$2007 \mathrm{MK}_{6}$ & 0.196 & 1.966 & 411 & 0.3 \\
$2010 \mathrm{VA}_{12}$ & 0.199 & 2.334 & 522 & 0.4 \\
$2015 \mathrm{DZ}_{53}$ & 0.196 & 2.828 & 679 & 0.2 \\
$2020 \mathrm{HD}_{9}$ & 0.195 & 4.237 & 1229 & 0.4 \\
$2020 \mathrm{KG}_{6}$ & 0.191 & 2.069 & 439 & 0.5 \\
\hline
\end{tabular}
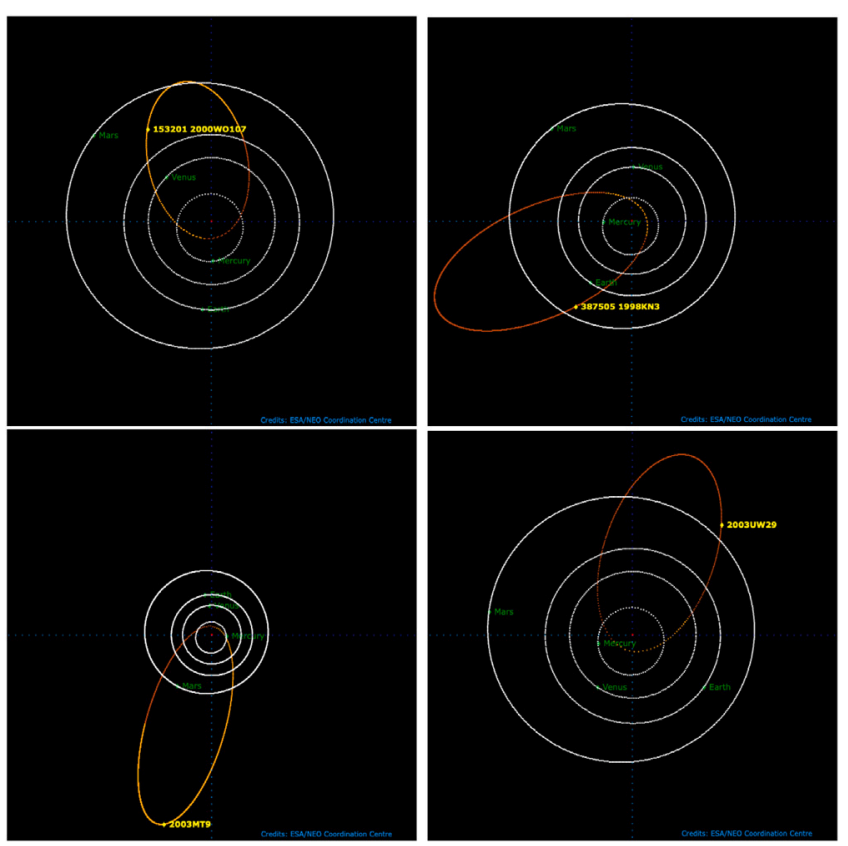

Fig. 2. Orbits as seen from the North Ecliptic Pole for asteroids (153 201) $2000 \mathrm{WO}_{107}$ [top left; 29], (387505) $1998 \mathrm{KN}_{3}$ [top right; 30], (455 426) $2003 \mathrm{MT}_{9}$ [bottom left; 31], and (506 491) $2003 \mathrm{UW}_{29}$ [bottom right; 32].

In both cases, ballistic tours combining fly-bys with the Earth (E) and Venus (V) are enough to achieve the desired science orbits. Parker Solar Probe is expected to perform seven Venus fly-bys to gradually reduce the periapsis to within 10 solar radii from the center of the Sun over about seven years of flight [36]. Solar Orbiter's cruise trajectory is based upon a fast EVVEV tour sufficient to reduce $q$ to less than 0.3 au in about two years from launch by exploiting resonances between the Earth and Venus [37].

However, the Icarus mission concept derives into somewhat more challenging requirements for the trajectory design. On the one hand, the identified asteroid candidates with small perihelion distances, while having perihelion distances comparable to those reached by the Parker Solar Probe and the Solar Orbiter, also have larger semimajor axes (i.e., orbital energy) than those of Parker Solar Probe's and Solar Orbiter's operational trajectories. On the other hand, the Icarus mission will not only require a specific orbit, but also a timely rendezvous with the target asteroid, ideally before its perihelion passage. Regarding this latter challenge, and as discussed in Section 5.3.1, a reasonable number of potential targets already exist, with the expectation that many more will be known by the time Icarus is launched. Hence, the specific final object to be targeted will ultimately depend on the launch date and relative phasing between planets and the targeted object. The remainder of the discussion instead focuses on the former challenge. That is, assessing the feasibility of accessing the targeted orbital region (i.e. relatively high semimajor axis with very small perihelion).

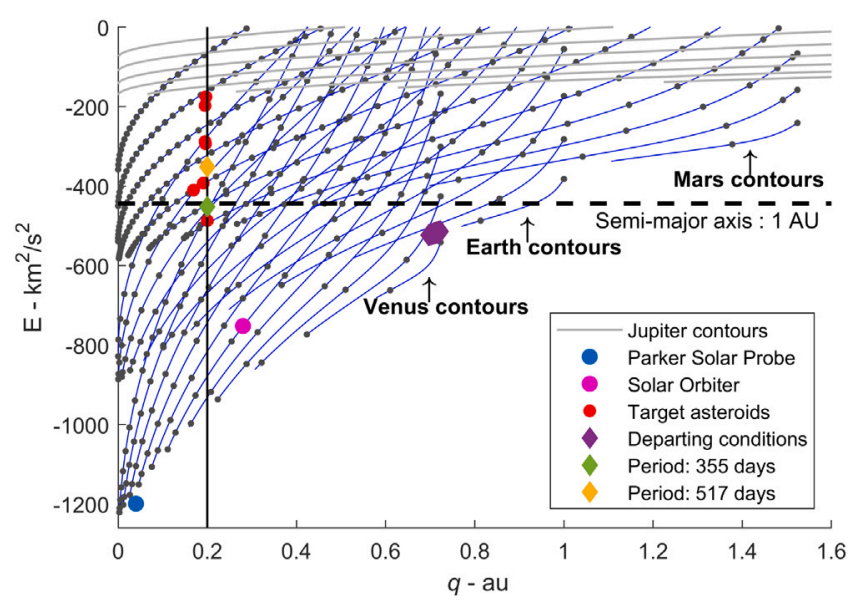

Fig. 3. Contours corresponding to different $v_{\infty}$ for Venus, Earth, Mars and Jupiter. The $v_{\infty}$ have discrete values of $1,5,9, \ldots \mathrm{km} / \mathrm{s}$ (increasing upwards). (For interpretation of the references to color in this figure legend, the reader is referred to the web version of this article.)

To assess the feasibility of reaching orbits with small perihelion distances and high orbital energies, complex MGA transfers are explored by means of a Tisserand graph-inspired search as described in [38]. Tisserand graphs have been widely used to efficiently tackle the combinatorial optimization problem posed by long MGA sequences, as this allows for fast computation of candidate fly-by tours with simple energy considerations [39]. This is particularly useful in early phases of mission design, when multiple trajectory options may be needed. Tisserand graphs can be obtained by parametrizing the Tisserand invariant with respect to the infinity velocity of the spacecraft relative to the gravity-assist body [40].

Fig. 3 shows a specific Tisserand $E, q$ graph to inform Icarus trajectory design [38]. In the graph, we describe the target orbital region of interest ( $q=0.2 \mathrm{au}$ ) as well as the set of possible departure conditions (Earth-Venus transfer configurations in 2032 and beyond). The graph also shows the contours of the velocity at infinity $\left(v_{\infty}\right)$ for Venus, Earth, Mars and Jupiter, which visualizes possible sequences of gravity assist to reach the target region of interest [39]. Thick marks along the $v_{\infty}$ contours indicate the maximum orbital change achievable in one single encounter with the given planet (as a result of a 200$\mathrm{km}$-altitude hyperbolic passage). However, Tisserand graphs contain no explicit information about planets position along their orbits. Therefore the phasing between the spacecraft and planet is ensured by means of a modified Tisserand graph exploration [38].

Two particular target orbits with $q=0.2$ au are highlighted in Fig. 3 (green and yellow diamonds). Examples of potential target asteroids in Table 4 are also shown in Fig. 3. The target marked with a green diamond has a period of 355 days $\left(E=-452 \mathrm{~km}^{2} / \mathrm{s}^{2}\right)$, which is already longer than that of asteroid (153201) $2000 \mathrm{WO}_{107}$. The target marked with a yellow diamond instead has a period of 517 days $(E=$ $-352 \mathrm{~km}^{2} / \mathrm{s}^{2}$ ), which implies an aphelion distance reaching the main asteroid belt. In general, the higher the energy along the $q=0.2$ au line, the higher the expected number of targets, according to the current best estimate of the orbit distribution of near-Earth asteroids [41]. Different combinations of planetary fly-bys and Deep Space Maneuvers (DSM) will allow for different transfer costs (in terms of total $\Delta V$ ), as well as different total transfer times (or time of flight; ToF). The methodology described in [38] allows to generate a pool of candidate MGA sequences that reach the desired target point on the graph.

The resultant Pareto front of MGA sequences that reach the green and yellow diamonds as well as asteroid (153201) $2000 \mathrm{WO}_{107}$ 's orbit, are shown in Fig. 4. Each diamond solution in Fig. 4 represents a complete sequence of planetary gravity assists terminating with a 


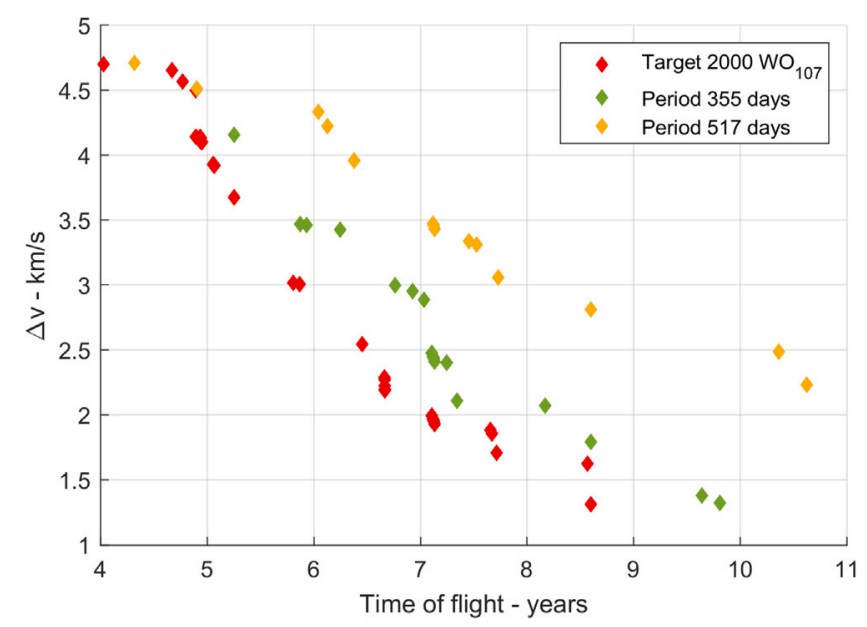

Fig. 4. The Pareto front of total $\Delta V$ and time of flight. (For interpretation of the references to color in this figure legend, the reader is referred to the web version of this article.)

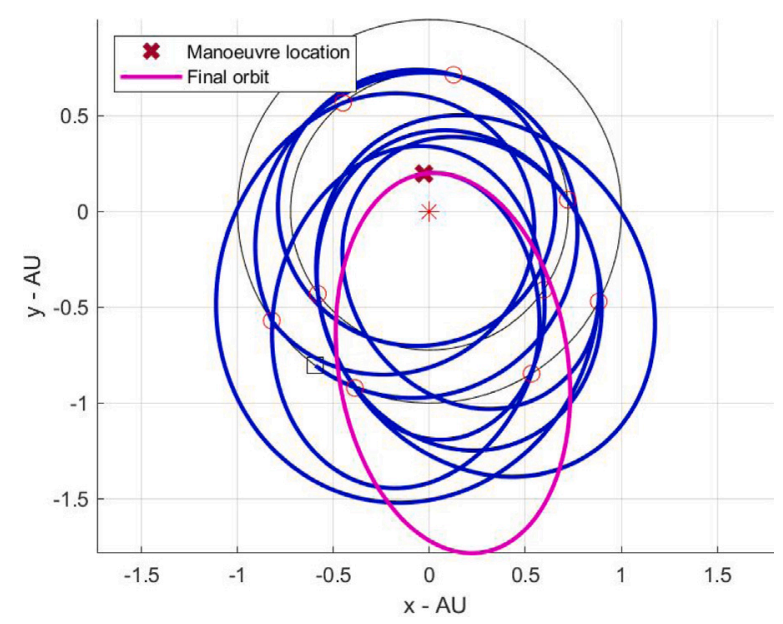

Fig. 5. MGA transfer to reach target orbit with $q=0.2$ au and a period of 355 days. The blue trajectory represents the cruise phase. The square represents the departing condition, while the circles represent planetary encounters. (For interpretation of the references to color in this figure legend, the reader is referred to the web version of this article.)

Hohmann-like transfer that allows to finally reach the target orbit. As it can be observed, a whole range of options exist, which follow a clear trend of decreasing $\Delta V$ at increasing time of flight. These results provide a preliminary understanding of the trajectory design space and inform the early iterations of the mission and system designs. Ultimately, the final transfer choice will need to be refined when specific asteroid targets, and launch and space segments are more clearly outlined. Nevertheless, these results show that while reaching orbits with $q=0.2$ au is clearly feasible, as also shown by past missions, achieving high energies (or aphelion distances) adds complexity to the transfer, which translate to long MGA sequences and long times of flight. As an example, Table 5 provides a summary of the best transfer option with less than 7.5 years of time of flight that reach the target orbits in Fig. 4. Note that the three examples in the table feature sequences of 9-11 planetary encounters in order reduce the transfer $\Delta V$ to manageable values. Lastly, Fig. 5 provides a heliocentric view of the complete sequence of planetary gravity assists and manoeuvres to reach an orbit with $q=0.2$ au and a period of 355 days.
Table 5

Summary of optimal transfer option available for a ToF $<7.5$ years.

\begin{tabular}{lllll}
\hline Target & MGA sequence & $\begin{array}{l}C_{3} \\
{\left[\mathrm{~km}^{2} / \mathrm{s}^{2}\right]}\end{array}$ & $\begin{array}{l}\Delta V \\
{[\mathrm{~km} / \mathrm{s}]}\end{array}$ & $\begin{array}{r}\text { ToF } \\
{[\mathrm{yrs}]}\end{array}$ \\
\hline 2000 $\mathrm{WO}_{107}$ & EVVEVVMEE & $<7.85$ & 1.9 & 7.1 \\
Period 355d & EVVEVEVVEVE & $<7.85$ & 2.1 & 7.2 \\
Period 517d & EVVEVVMEE & $<7.85$ & 3.3 & 7.1 \\
\hline
\end{tabular}

Table 6

Worst-case solar flux based on measurements at $r=1$ au and extrapolated to $r=0.2$ au [42].

\begin{tabular}{llll}
\hline Type & $\begin{array}{l}\text { Wavelength } \\
{[\mathrm{nm}]}\end{array}$ & $\begin{array}{l}\text { Flux at } 1 \text { au } \\
\mathrm{W} / \mathrm{m}^{2}\end{array}$ & $\begin{array}{l}\text { Flux at } 0.2 \text { au } \\
\mathrm{W} / \mathrm{m}^{2}\end{array}$ \\
\hline Near UV & $180-400$ & 177 & 4425 \\
UV & $<180$ & 0.046 & 1.15 \\
UV & $100-150$ & 0.015 & 0.375 \\
EUV & $10-100$ & 0.004 & 0.1 \\
X-ray & $1-10$ & 0.0001 & 0.0025 \\
Flare X-ray & $0.1-1$ & 0.001 & 0.025 \\
\hline
\end{tabular}

Table 7

Typical solar wind parameters at heliocentric distances $r=0.2$ au and $r=1$ au [42].

\begin{tabular}{lll}
\hline Parameter & $1 \mathrm{au}$ & $0.2 \mathrm{au}$ \\
\hline$\rho\left[\mathrm{cm}^{-3}\right]$ & 8.3 & 217.5 \\
$v[\mathrm{~km} / \mathrm{s}]$ & 468 & 468 \\
$T_{e}[\mathrm{~K}]$ & $1.0 \times 10^{5}$ & $1.0 \times 10^{5}$ \\
$\lambda_{D}[\mathrm{~m}]$ & 7.3 & 1.48 \\
\hline
\end{tabular}

\subsection{Mission environment overview}

We will next look at the mission environment. All values used are based on the environmental analysis conducted during the BepiColombo and the Parker Solar Probe missions, and adjusted using the inverse square law. Both missions provide a good starting point for analysis due to their similar mission profiles.

\subsubsection{Electromagnetic radiation}

The spacecraft will be subjected to a variety of electromagnetic radiation during its journey. The direct solar flux, present during the entire mission, will be the biggest factor. The solar irradiance varies from $350 \mathrm{~W} / \mathrm{m}^{2}$ at $r=1.5 \mathrm{au}$ to $35000 \mathrm{~W} / \mathrm{m}^{2}$ at $r=0.2 \mathrm{au}$. The spacecraft can also be subjected to planetary infrared (IR) radiation which can vary greatly even over small time scales [42]. For example, in the case of Venus the geometric albedo in the visible wavelengths is 0.65 by average and the solar irradiance at the distance of Venus is about $2600 \mathrm{~W} / \mathrm{m}^{2}$. Hence the irradiation reflected off of Venus is about $1700 \mathrm{~W} / \mathrm{m}^{2}$.

The solar radiation spectrum is approximated by a black-body radiation curve $(T=5780 \mathrm{~K})$ which is the basis for the measured worst-case-scenario values at $r=1$ au (Table 6). The UV radiation analysis is particularly important since it is known to degrade the surface materials of a spacecraft causing a variety of problems [43].

\subsubsection{Plasma environment}

The relevant plasma environments include the incoming solar wind and planetary magnetospheres during orbital maneuvers. The solar wind speeds range from $300 \mathrm{~km} / \mathrm{s}$ to $1200 \mathrm{~km} / \mathrm{s}$, but are most commonly around $470 \mathrm{~km} / \mathrm{s}$. They carry ions with considerable kinetic energy ( $1 \mathrm{keV}$ for protons and $4 \mathrm{eV}$ for He-ions) that can cause sputtering that degrades the spacecraft surface [42]. Typical solar wind parameters are summarized in Table 7.

The natural plasma environment around the spacecraft can change due to emitted gas. For example, the produced neutral gas, caused by sputtering, can, in turn, produce a low-energy $(<10 \mathrm{eV})$ ion population, ionized by sunlight, near the spacecraft [42]. These ions may be drawn to negatively charged surfaces changing the optical properties of the components. There also exists a possibility for harmful electrostatic discharges from high-voltage components. 


\subsubsection{High-energy particles}

High-energy particles such as electrons $(\sim 100 \mathrm{keV})$ and ions $(\sim$ $1 \mathrm{MeV}$ ) can easily penetrate spacecraft walls and deposit a considerable radiation dosage to critical components [42]. Absorbed ions can develop single-event upsets (SEU), where a transistor changes state due to a specific energy transfer, causing considerable damage to the memory components of the spacecraft. Furthermore, high-energy particles carry significant kinetic energy and dislodge particles from materials, which then can exhibit altered electrical, mechanical, and optical properties.

Most high-energy electrons, encountered by the spacecraft, are either from solar activity or from the Jovian system, if solar activity is low. The electrons from the Jovian system are fairly energetic, with maximum energies of $\sim 5 \times 10^{4} \mathrm{keV}$, but have low fluxes $(\sim$ $10^{-10}-10^{-1} \mathrm{~cm}^{-2} / \mathrm{sr} / \mathrm{s} / \mathrm{keV}$ ) while solar electrons have similar maximum energy values $\left(\sim 2 \times 10^{4} \mathrm{keV}\right)$ but much higher flux values $(\sim$ $\left.2.5 \times 10^{-2}-2.5 \times 10^{8} \mathrm{~cm}^{-2} / \mathrm{sr} / \mathrm{s} / \mathrm{keV}\right)$. In addition, during periods of high solar activity, particle events have been recorded to produce protons with energies higher than $100 \mathrm{MeV}$ [42]. As the 25th solar cycle started between 2019 and 2020, with expected peak activity around 2025, we can estimate that the 26 th solar cycle could start somewhere in the first half of 2030 and have its peak activity in the latter half of 2030 [44]. This might overlap with Icarus' closest approach to the Sun and should be considered in later iterations of the mission concept.

\subsubsection{Micrometeoroid and dust environment}

A collision with a micrometeoroid can cause serious damage depending on its speed, impact angle, weight, and size. Particles with diameters $<1 \mathrm{~mm}$ can degrade surface materials and cause damage to optical components while larger ones can damage or destroy spacecraft structures.

According to the analysis done for the Parker Solar Probe mission, the dust density in ecliptic orbits (latitude $\pm 30^{\circ}$ ) around the Sun is inversely proportional to the heliocentric distance for $0.05 \mathrm{au}<r<$ $1 \mathrm{au}$. The typical bulk density is, however, $\sim 2.5 \mathrm{~g} / \mathrm{cm}^{3}$ [45]. These streams can have relative velocities up to $350 \mathrm{~km} / \mathrm{s}$ and can cause serious damage to the spacecraft. Further analysis on the particulate size and fluence is needed to estimate the order of magnitude of impact probabilities and the size of spacecraft shielding.

\section{Space segment}

The main challenge for the Icarus mission is to design a space segment that is as simple and cost effective as possible. The preliminary analysis was conducted in a study using conservative assumptions and estimations utilizing concept design margins ( $+20 \%$ to budgets) where applicable. The following design is the first iteration of the space segment and serves as a proof of concept. It relies on heritage from previous ESA (BepiColombo and Rosetta) and NASA (Parker Solar Probe, Deep Impact, and Messenger) missions.

The preliminary analysis shows that the proposed space segment is technologically feasible. This means that no new technology is needed and all existing hardware is flight proven. Icarus is also compliant with ESA F1 mission constraints with the cost budget and the trajectory being the only aspects requiring a more in-depth analysis.

\subsection{Spacecraft system}

Fig. 6 shows the spacecraft in both launch and operational configurations. The spacecraft will deploy two solar arrays, symmetrically on the opposite sides, that can be actuated for Sun tracking and to limit the solar input. For heat protection Icarus will use a heat shield that faces the Sun during the perihelion passage. Located on the opposite side of the heat shield is a steerable high-gain antenna (HGA) actuated by a dual-gimbal mechanism with two degrees of freedom. The payload suite has unobscured view of the asteroid while allowing the solar array and the heat shield to face the Sun. A set of $10 \mathrm{~N}$ bi-propellant thrusters

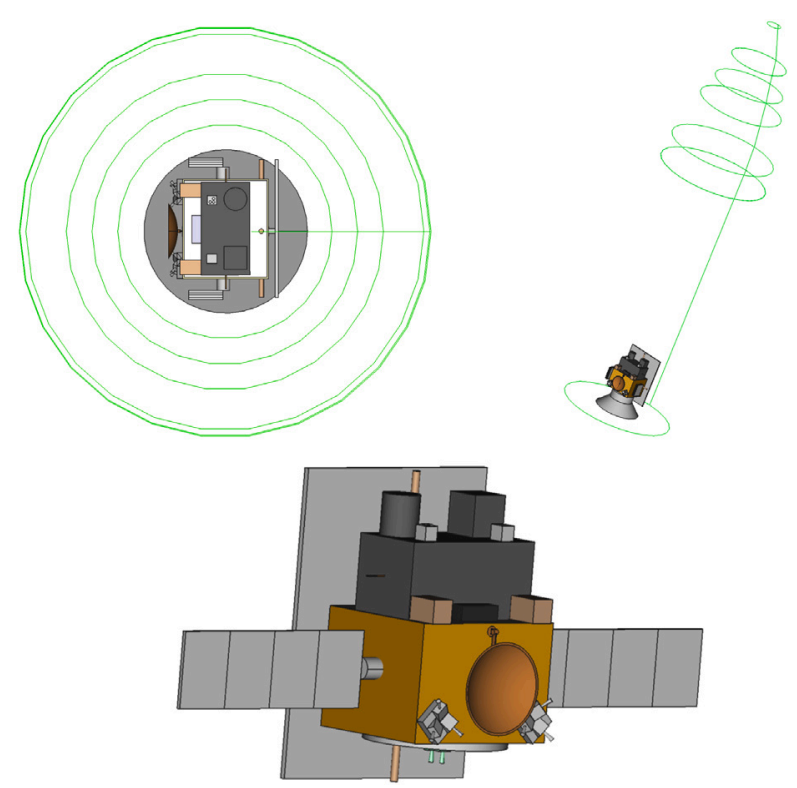

Fig. 6. The spacecraft in launch and operational configuration. The Ariane 62 internal volume is used for Ref. [46].

execute all major orbital maneuvers while two sets of small-Newton thrusters provide 3 -axis attitude control and help with reaction wheel desaturation. The four propellant tanks, located inside the spacecraft hull, distribute bi-propellant composed of mixed oxides of nitrogen (MON) and monomethylhydrazine (MMH) to all thrusters. Aluminum alloy (Al 6061) is proposed for the structures due to its strength and thermal properties. A shield thickness of approximately $8.5 \mathrm{~mm}$ is also needed for estimated micrometeoroid environment (Section 5.4). The structure is reinforced with truss supports capable of bearing the launch loads. Surrounding the main engines, a payload adaptor ring bridges the launcher and the spacecraft by connecting to the payload adaptor fitting. The payload adaptor fitting, used here as an example, is part of a payload adaptor system provided by Ariane Space and is designed to fit a spacecraft into their Ariane 62 launch vehicle [46].

The spacecraft has an estimated wet mass of about $880 \mathrm{~kg}$ including the concept design margin. The mission payload weighs around $47 \mathrm{~kg}$ not including any additional instrumentation [22,23,26,27]. The total estimated propellant budget is around $530 \mathrm{~kg}$ with $470 \mathrm{~kg}$ for the main propulsion and $60 \mathrm{~kg}$ for the reaction control system. The propellant budget is calculated for a $2.7 \mathrm{~km} / \mathrm{s}$ transfer, including the concept design margin, despite the preliminary $\Delta V$ analysis in Section 5.3.2 (see further discussion in Section 6.2). The launch adaptor is not included in the mass budget since it is assumed to be included in the ESA launch costs. It would, however, add only a maximum of $50 \mathrm{~kg}$ to the overall mass budget.

Icarus will rely on a thermal heat shield for protection near the Sun. The heat shield, heritage to the Parker Solar Probe [45], is constructed of carbon-to-carbon (C-C) composite shell containing a layer of reticulated vitreus carbon (RVC) foam. The RVC is the shield's main insulator with thickness determined by the surface temperature and the allowed heat flow through the insulator and into the spacecraft. The Sun-facing side of the shield has a coating, such as alumina $\left(\mathrm{Al}_{2} \mathrm{O}_{3}\right)$, that will maximize reflectivity and minimize absorptivity. The shield was proven functional for the purposes of Icarus at the end of 2019 when the Parker Solar Probe reached $r<0.2$ au. The outside of the Icarus spacecraft is wrapped in Kapton multi-layer insulation (MLI) film while possible patch heaters, heat piping, and radiator panels ensure that vital components remain within acceptable thermal limits.

The power for the spacecraft is produced by two actuated solar arrays with one degree of freedom. The array wings can be rotated for 
Sun tracking and to reduce incoming solar power near the perihelion. The proposed panels bear heritage to the MESSENGER mission [47] utilizing optical solar reflector (OSR) mirrors and solar cells with a ratio of 2:1 to reduce panel absorbance. Similar solar panel architecture was also used aboard the Parker Solar Probe [45] and the Solar Orbiter [48]. The array panels are sized according to the lowest solar irradiance, which happens when the spacecraft is the farthest from the Sun. As a baseline this is estimated to be $r \sim 1.5 \mathrm{au}$. The size of the arrays may need to be adjusted after target selection when the mission design is known in detail. The system also includes a battery assembly which will provide power during early operations, before solar array deployment, and keep the nominal systems running in the case of an eclipse.

The attitude and orbit control system (AOCS) together with the guidance, navigation and control (GNC) subsystem ensures correct spacecraft attitude, orientation, and location in space. The Icarus AOCS/GNC includes two star trackers and four solar-horizon sensors [heritage to the Parker Solar Probe mission; 45] for triangulating spacecraft location and orientation related to the Sun, two inertial measurement units to recognize the spacecraft's attitude, and four reaction wheel assemblies and two sets of small-Newton thrusters for attitude control. When approaching the perihelion, the attitude control becomes a high priority in order to remain in the shadow of the heat shield. The allowed pointing error to the Sun is related to the size of the shield, and can be increased with relatively small changes. When approaching the target, the main telescope can be used for visual tracking and the fine pointing achieved by utilizing the reaction wheels. A similar system configuration has already been used in previous smallbody missions such as Rosetta and Deep Impact. The FOV of the payload will also allow for some pointing error that depends on the distance to the target.

An early version of the Icarus propulsion subsystem contains a set of $10 \mathrm{~N}$ thrusters, that provide enough thrust during the cruise phase of the mission to reach the needed trajectory and to intercept the target asteroid. Relying on a design with a long list of heritage, such as BepiColombo and Rosetta [49,50], Icarus will deploy at least three $10 \mathrm{~N}$ bi-propellant thrusters with a maximum thrust of $12.5 \mathrm{~N}$ each and specific impulse $\left(I_{s p}\right)$ of about $290 \mathrm{~s}$ [51]. The whole propulsion system includes all the required components including high-pressure regulators, flow control units, sensors, and piping. The thrusters are designed to work in both long-term steady state and pulse-mode operation.

The communications subsystem will have to be able to reliably receive commands and updates from ground and transmit large amounts of scientific data. The Icarus antenna system is modeled after the Parker Solar Probe mission [45]. It has an actuated high-gain antenna (HGA) capable of transmitting in Ka-band and receiving in X-band while two hard-mounted low-gain antennas (LGA) transmit and receive only in X-band. The LGA system works during early operations and as a backup system. The spacecraft will produce a large amount of data and thus the on-board data handling (OBDH) system will need to be able to store large amounts of data reliably. The BepiColombo OBDH system provides a large capacity solid-state mass memory device $(\sim 384 \mathrm{~Gb})$ as well as a SpaceWire interface capable of handling large bitrates [49]. During science operations all the collected and stored data from spacecraft's internal hard drive will be transmitted via the HGA in Ka-band and received, most probably, by the ESA Tracking Station Network's (ESTRACK) $35 \mathrm{~m}$ Deep Space Tracking Stations in Cebreros, New Norcia or Malargüe. Considering heritage from both the Rosetta and the Parker Solar Probe missions and estimating an average downlink speed of $100 \mathrm{kbps}$ from 1.5 AU in Ka-band, with an effective downlink time (dump duration) of $16 \mathrm{~h}$ per day, around $175 \mathrm{~Gb}$ of science data from Icarus' internal memory could be transmitted within 40 days [45,52]. The effective downlink time will, however, depend on the final mission profile and ground station pass durations. Icarus is also estimated to produced a constant $1 \mathrm{kbps}$ of housekeeping data.

The cost of the mission was estimated using an analogy cost estimation methodology which provides a rough magnitude of the budget and serves as a good starting point for further analysis. The estimate complies with ESA F-class mission constraints and does not disqualify the Icarus mission.
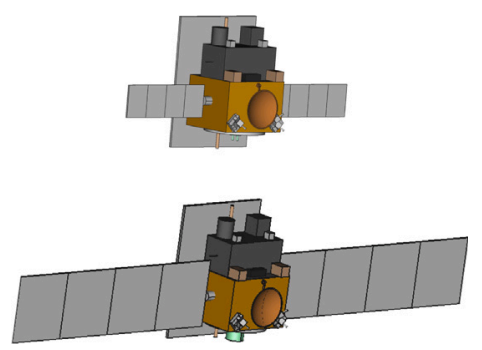

Fig. 7. The Icarus spacecraft using chemical propulsion (top) and electrical propulsion (bottom).

\subsection{Technical challenges and alternative architectures}

A preliminary trajectory is depicted in Section 5.3 .2 (Fig. 5) featuring a total $\Delta V$ of about $2.1 \mathrm{~km} / \mathrm{s}$ and a time of flight of 7.2 years. However, the Pareto front solutions presented in Fig. 4 demonstrate the existence of both solutions with smaller $\Delta V$ or shorter time of flight requirements. The baseline system design presented in Section 6.1 considered a chemical propulsion system with a $\Delta V$ capability of $2.7 \mathrm{~km} / \mathrm{s}$. With such a $\Delta V$ budget a heliocentric cruise of 6.5 years would be possible, which would correspond to a mission duration of seven years, considering six months of science operations.

If a larger $\Delta V$ turns out to be necessary, to shorten the mission duration, for example, or if the aforementioned $\Delta V$ budgets are ultimately not considered feasible with chemical propulsion, Icarus may need to rely on a low-thrust propulsion system. One option is the electric propulsion system flown aboard the BepiColombo spacecraft. It includes four Kaufman-type ion-engines with a maximum thrust of approximately $150 \mathrm{mN}$ each and specific impulse of about $4300 \mathrm{~s} \mathrm{[49].}$ A low-thrust propulsion system comes with its own set of problems such as high power demands and overall system complexity. Using just one of BepiColombo's ion-engines and scaling it down would increase the total power requirement for Icarus up to $3 \mathrm{~kW}$ which would, in turn, require a significantly larger area for the solar arrays (see comparison in Fig. 7). Preliminary analysis shows that even with a low-thrust propulsion system and associated design changes, the mission would still stay within the mass budget of an ESA F-class mission. It should also be mentioned that most of the reference solutions described in Fig. 4 allow sufficient coasting time to perform the necessary lowthrust $\Delta V$ maneuvers, hence indicating the feasibility to refine a similar transfer with an adequate control law for the continuous acceleration case.

If the mission will opt for a low-thrust system it could benefit from an external propulsion system, which is ejected after its functionality has expired. A similar architecture is used in BepiColombo and was also considered for the Solar Orbiter $[48,49]$. A configuration like this could solve the problem of excess heat leaking into the spacecraft, but would in turn increase the complexity of the system. If, however, this could be designed to be a cost-effective solution, it would greatly increase the likelihood of a successful mission.

The Icarus AOCS/GNC subsystem currently utilizes four sets of reaction wheel assemblies for attitude control. The system also includes a reaction control system in order to counteract momentum stored by the reaction wheels. Depending on the final configuration of the spacecraft and allowed slewing rate, a set of smaller wheels with less momentum storage might prove more suitable. With smaller reaction wheels, a cold gas reaction control system could also be implemented, simplifying the design even further.

In addition to resizing the solar arrays, the mission could benefit from a different panel architecture all together. The main downside of the MESSENGER panels is their weight and cost, and as Icarus does not really need thermal protection until $r \sim 0.4$ au, it could thus utilize more conventional solar panels until then. The challenge is to 
design a system where the switch between the two kinds of panels happen. One option is an architecture where only a fraction of the array includes MESSENGER panels, while the remaining portion would have normal panels. This would require some kind of protection of the nonreflective array area near the perihelion to counter for overheating. The spacecraft could also utilize a system where the heat shield is used to house the MESSENGER panels while the array, with normal panels, is actuated to functionally disable them.

As mentioned in Section 2, targets with perihelion distances $q<$ 0.2 au might prove to be preferable. This should be possible with only slight modifications to the Icarus system, and can be explored more in the following iterations. However, as the heat flux and radiation increases exponentially with smaller $q$, a suitable trade-off with system complexity and perihelion distance has to be found.

\section{Conclusions}

We presented the science case for a rendezvous mission to an asteroid, that has a perihelion distance of $\sim 0.2 \mathrm{au}$, and described the scientifically interesting measurements that would have to be obtained during the asteroid's perihelion passage to meet the science objectives. We then laid out a concept for a mission, Icarus, that could obtain those measurements.

The preliminary Icarus design presented is a proof-of-concept to determine if the mission is feasible considering ESA F-class constants and existing flight proven technology. Icarus is a novel space mission in that it combines two challenging mission profiles-an asteroid rendezvous at a small heliocentric distance. By deploying at least three instruments and a robust architecture, Icarus will reliably and cost effectively complete its mission and provide unique measurements that cannot be obtained from the Earth.

We conclude that there exists no technological bottle-necks and all the chosen hardware have TRL 9. The payload suite includes one instrument which is currently in development, but has a TRL 5/6 with solid heritage behind it. With conservative assumptions and estimations the Icarus spacecraft is also in compliance with most of the ESA Fclass mission requirements and constraints set in the 2018 call for proposals, including weight and cost. However, due to the challenging mission trajectory and predicted cruise time, we must note that the Icarus proposal may only be marginally feasible as an F-class mission and would require the M5 mission, with which the F2 mission will be launched, to have a similar trajectory profile. EnVision would be an obvious candidate sharing additional $3 \mathrm{~km} / \mathrm{s}$ escape velocity. Despite this we would like to emphasize that the Icarus mission would most certainly be feasible as a larger mission and expanded budgets would even allow for additional science to be conducted in, for example, nearSun solar studies. Thus, if EnVision is not selected, there are a few alternatives going forward: (i) perform a more in-depth mission analysis to see if Icarus trajectory and budgets allow Theseus as a launch companion, (ii) expand the mission to $\mathrm{M}$ class with more scientific output, or (iii) wait for the F3 mission call.

Target selection is the most critical issue for the entire mission because it dictates the required $\Delta V$ budget. While asteroid Phaethon would be a scientifically interesting target, it is challenging to find a mission design that can accommodate its small $q$ and large $Q$ given the F-class constraints on mass and mission duration. However, upcoming surveys such as LSST will guarantee that there will be ample targets to choose from in the years to come. Proposing Icarus as an M-class mission would also allow asteroid Phaethon to be considered.

There is still a lot of work to be done, especially in spacecraft design, instrument design, and the mission profile, but we have not identified any critical aspects that would make the Icarus mission unfeasible for scientific, technological, financial, or programmatic reasons. Hence we hope that our work will trigger wider interest and further studies focusing on topics relevant to Icarus (or an Icarus-like mission) in the space engineering and planetary science communities.

\section{Declaration of competing interest}

The authors declare that they have no known competing financial interests or personal relationships that could have appeared to influence the work reported in this paper.

\section{Acknowledgments}

This work was supported by the Academy of Finland, the Waldemar von Frenckell foundation, Finland, and the Knut and Alice Wallenberg Foundation, Sweden.

\section{References}

[1] P. Farinella, C. Froeschlé, C. Froeschlé, R. Gonczi, G. Hahn, A. Morbidelli, G.B. Valsecchi, Asteroids falling into the Sun, Nature 371 (6495) (1994) 314-317.

[2] M. Granvik, A. Morbidelli, R. Jedicke, B. Bolin, W.F. Bottke, E. Beshore, D. Vokrouhlický, M. Delbò, P. Michel, Super-catastrophic disruption of asteroids at small perihelion distances, Nature 530 (2016) 303-306.

[3] M. Granvik, A. Morbidelli, R. Jedicke, B. Bolin, W.F. Bottke, E. Beshore, D. Vokrouhlický, D. Nesvorný, P. Michel, Debiased orbit and absolute-magnitude distributions for near-Earth objects, Icarus 312 (2018) 181-207.

[4] H.H. Hsieh, D. Jewitt, A population of comets in the Main Asteroid Belt, Science 312 (5773) (2006) 561-563.

[5] M. Küppers, L. O’Rourke, D. Bockelée-Morvan, V. Zakharov, S. Lee, P. von Allmen, B. Carry, D. Teyssier, A. Marston, T. Müller, J. Crovisier, M.A. Barucci, R. Moreno, Localized sources of water vapour on the dwarf planet (1)Ceres, Nature 505 (7484) (2014) 525-527.

[6] D. Jewitt, J. Li, J. Agarwal, The dust Tail of Asteroid (3200) Phaethon, Astrophys. J. Lett. 771 (2) (2013) L36.

[7] M.M. Knight, A. Fitzsimmons, M.S.P. Kelley, C. Snodgrass, Comet 322P/SOHO 1: An asteroid with the Smallest Perihelion distance?, Astrophys. J. Lett. 823 (1) (2016) L6.

[8] D. Jewitt, H. Hsieh, Physical observations of 2005 UD: A Mini-Phaethon, Astron. J. 132 (4) (2006) 1624-1629.

[9] M. Devogèle, E. MacLennan, A. Gustafsson, N. Moskovitz, J. Chatelain, G. Borisov, S. Abe, T. Arai, G. Fedorets, M. Ferrais, M. Granvik, E. Jehin, L. Siltala, M. Pöntinen, M. Mommert, D. Polishook, B. Skiff, P. Tanga, F. Yoshida, New evidence for a Physical link between Asteroids (155140) 2005 UD and (3200) Phaethon, Planet. Sci. J. 1 (1) (2020) 15.

[10] ESA, F Mission Call - Technical Annex (ESA-SCI-F-ESTEC-TN-2018-003), ESTEC (ESA), Noordwijk, Netherlands, 2018.

[11] M. Delbo, G. Libourel, J. Wilkerson, N. Murdoch, P. Michel, K.T. Ramesh, C. Ganino, C. Verati, S. Marchi, Thermal fatigue as the origin of regolith on small asteroids, Nature 508 (7495) (2014) 233-236.

[12] D. Jewitt, The active asteroids, Astron. J. 143 (3) (2012) 66.

[13] D. Vokrouhlický, W.F. Bottke, S.R. Chesley, D.J. Scheeres, T.S. Statler, The Yarkovsky and YORP effects, in: Asteroids IV, 2015, pp. 509-531, http://dx. doi.org/10.2458/azu_uapress_9780816532131-ch027.

[14] J.K. Steckloff, S.A. Jacobson, The formation of striae within cometary dust tails by a sublimation-driven YORP-like effect, Icarus 264 (2016) 160-171.

[15] O. Poch, A. Pommerol, B. Jost, N. Carrasco, C. Szopa, N. Thomas, Sublimation of water ice mixed with silicates and tholins: Evolution of surface texture and reflectance spectra, with implications for comets, Icarus 267 (2016) 154-173.

[16] P. Wiegert, P. Brown, P. Pokorný, Q. Ye, C. Gregg, K. Lenartowicz, Z. Krzeminski, D. Clark, Supercatastrophic disruption of asteroids in the context of SOHO comet, fireball, and meteor observations, Astron. J. 159 (4) (2020) 143.

[17] P. Brown, P. Wiegert, D. Clark, E. Tagliaferri, Orbital and physical characteristics of meter-scale impactors from airburst observations, Icarus 266 (2016) 96-111.

[18] A. Morbidelli, M. Delbo, M. Granvik, W.F. Bottke, R. Jedicke, B. Bolin, P. Michel, D. Vokrouhlický, Debiased albedo distribution for Near Earth Objects, 2020, arXiv e-prints arXiv:2001.03550.

[19] A.S. Rivkin, H. Campins, J.P. Emery, E.S. Howell, J. Licandro, D. Takir, F. Vilas, Astronomical observations of volatiles on asteroids, in: Asteroids IV, 2015, pp. 65-87, http://dx.doi.org/10.2458/azu_uapress_9780816532131-ch004.

[20] K.J. Graves, D.A. Minton, J.L. Molaro, M. Hirabayashi, Resurfacing asteroids from thermally induced surface degradation, Icarus 322 (2019) 1-12.

[21] S. Greenstreet, H. Ngo, B. Gladman, The orbital distribution of Near-Earth Objects inside Earth's orbit, Icarus 217 (1) (2012) 355-366.

[22] H.U. Keller, C. Barbieri, P. Lamy, H. Rickman, R. Rodrigo, K.P. Wenzel, H. Sierks, M.F. Ahearn, F. Angrilli, M. Angulo, M.E. Bailey, P. Barthol, M.A. Barucci, J.L. Bertaux, G. Bianchini, J.L. Boit, V. Brown, J.A. Burns, I. Büttner, Osiris - the scientific camera system on-board rosetta, Space Sci. Rev. 128 (2007) 433-506.

[23] T. Kohout, A. Näsilä, T. Tikka, M. Granvik, A. Kestilä, A. Penttilä, J. Kuhno, K. Muinonen, K. Viherkanto, E. Kallio, Feasibility of asteroid exploration using CubeSats-ASPECT case study, Adv. Space Res. 62 (2018) 2239-2244. 
[24] H. Saari, V.-V. Aallos, A. Akujärvi, T. Antila, C. Holmlund, U. Kantojärvi, J. Mäkynen, J. Ollila, Novel miniaturized hyperspectral sensor for UAV and space applications, in: R. Meynart, S.P. Neeck, H. Shimoda (Eds.), Sensors, Systems, and Next-Generation Satellites XIII, Vol. 7474, International Society for Optics and Photonics, SPIE, 2009, pp. 517-528, http://dx.doi.org/10.1117/12.830284.

[25] A. Näsilä, C. Holmlund, R. Mannila, I. Näkki, H.J. Ojanen, A. Akujärvi, H. Saari, D. Fussen, D. Pieroux, P. Demoulin, PICASSO VISION instrument design, engineering model test results, and flight model development status, in: A. Comerón, E.I. Kassianov, K. Schäfer (Eds.), Remote Sensing of Clouds and the Atmosphere XXI, Vol. 10001, International Society for Optics and Photonics, SPIE, 2016, pp. 50-63, http://dx.doi.org/10.1117/12.2241956.

[26] S. Jones, N. Paschalidis, M. Rodriguez, E.C. Sittler, D.J. Chornay, P. Uribe, T. Cameron, A compact ion and neutral mass spectrometer for measuring atmospheric composition with preliminary results from the dellingr mission, in: American Geophysical Union, Fall Meeting, Vol. 2017, 2017, pp. A41I-2413.

[27] A. Wolf, R. Laufer, G. Lightsey, G. Herdrich, R. Srama, H.P. Röser, T. Hyde, Piezo dust detector (PDD) - A modular miniaturized in-situ measurement instrument for dust research, in: 43rd Lunar and Planetary Science Conference, 2012, p. 2136.

[28] P. Vereš, S.R. Chesley, High-fidelity simulations of the Near-Earth Object Search Performance of the Large Synoptic survey telescope, Astron. J. 154 (1) (2017) 12.

[29] ESA, NEODyS-2 (153201) 2000WO107, 2000, https://newton.spacedys.com/ neodys/index.php?pc=1.1.0\&n=153201. Accessed: 2021-01-01.

[30] ESA, NEODyS-2 (387505) 1998KN3, 1998, https://newton.spacedys.com/ neodys/index.php?pc=1.1.0\&n=1998+KN3. Accessed: 2019-11-25.

[31] ESA, NEODyS-2 (455426) 2003MT9, 2003, https://newton.spacedys.com/ neodys/index.php?pc=1.1.0\&n=2003+MT9. Accessed: 2021-01-01.

[32] ESA, NEODyS-2 (506491) 2003UW29, 2003, https://newton.spacedys.com/ neodys/index.php?pc=1.1.0\&n=506491. Accessed: 2021-01-04.

[33] Y. Guo, Solar probe plus: Mission design challenges and trades, Acta Astronaut. 67 (9-10) (2010) 1063-1072.

[34] D. McComas, L. Acton, M. Balat-Pichelin, V. Bothmer, R. Dirling, Solar Probe Plus: Report of the Science and Technology Definition Team, Natl. Aeronaut. Sp. Adm. Goddard Sp. Flight Center, Greenbelt, Maryl., 2008.

[35] N. Rando, L. Gerlach, G. Janin, B. Johlander, A. Jeanes, A. Lyngvi, R. Marsden, A. Owens, U. Telljohann, D. Lumb, T. Peacock, Solar Orbiter assessment study and model payload, in: S. Fineschi, R.A. Viereck (Eds.), Solar Physics and Space Weather Instrumentation, Vol. 5901, International Society for Optics and Photonics, SPIE, 2005, pp. 244-254, http://dx.doi.org/10.1117/12.621790.

[36] P. Thompson, T. Goodson, M. Chung, D. Jones, E. Lau, N. Mottinger, P. Valerino, Parker solar probe navigation: One year from launch, in: Proceedings of the 2017 AAS/AIAA Astrodynamics Specialists Conference AAS-17-604, 2017.
[37] J. Sanchez Perez, W. Martens, G. Varga, Solar orbiter 2020 february mission profile, Adv. Astronaut. Sci. 167 (2018) 1395-1410.

[38] A. Bellome, J.-P.S. Cuartielles, L. Felicetti, S. Kemble, Modified Tisserand Map Exploration for Preliminary Multiple Gravity Assist Trajectory Design, IAFASTRO, 2020 .

[39] N. Strange, J. Longuski, Graphical method for gravity-assist trajectory design, J. Spacecr. Rockets 37 (2002) 9-16.

[40] J. Miller, C. Weeks, Application of Tisserand's criterion to the design of gravity assist trajectories, in: AIAA/AAS Astrodynamics Specialist Conference and Exhibit, 2002, p. 4717.

[41] M. Granvik, A. Morbidelli, R. Jedicke, B. Bolin, W. Bottke, E. Beshore, D. Vokrouhlický, D. Nesvorný, P. Michel, Debiased orbit and absolute-magnitude distributions for near-earth objects, Icarus 312 (2018) 181-207.

[42] J. Sørensen, H. Evans, Mercury Environmental Specification (Part II) BepiColombo Definition Study (SCI-PF/BC/TN/02), ESTEC (ESA), Noordwijk, Netherlands, 2004.

[43] A. Stiegman, L. R., Ultraviolet and vacuum-ultraviolet radiation effects on spacecraft thermal control materials [nato ASI series], Behav. Syst. Space Environ. 245 (1993) 259-266.

[44] S. McIntosh, S. Chapman, R. Leamon, R. Egeland, N. Watkins, Overlapping magnetic activity cycles and the sunspot number: Forecasting sunspot cycle 25 amplitude, Sol. Phys. 295 (163 (2020)) (2020).

[45] NASA, Solar Probe Plus: Report of the Science and Technology Definition Team (NASA/TM-2008-214161), Goddard Space Flight Center (NASA), Greenbelt, Maryland, 2008

[46] Arianespace, Ariane 6 User's Manual - Issue 1 Revision 0, Arianespace, Ariane Group, 2018.

[47] G. Dakermanji, J. Jenkins, C.J. Ercol, The messenger spacecraft solar array design and early mission performance, 2006 IEEE 4th World Conference on Photovoltaic Energy Conference, 2006.

[48] N. Rando, L. Gerlach, G. Janin, B. Johlander, A. Jeanes, A. Lyngvi, R. Marsden, A. Owens, U. Telljohann, D. Lumb, T. Peacock, Solar Orbiter assessment study and model payload, Proc. SPIE 5901 (2005) 59010X-1.

[49] C. Steiger, E. Montagnon, A. Accomazzo, Flight Operations Preparation for the BepiColombo Mission to Mercury: Concepts and Challenges, ESTOC (ESA), Darmstadt, Germany, 2016.

[50] D. Stramaccioni, The Rosetta propulsion system, in: 4th International Spacecraft Propulsion Conference, in: ESA Special Publication, vol. 555, 2004, p. 3.1.

[51] Arianegroup, 10N, 200N, 400N - Chemical Bi-Propellant Thruster Family, Ariane Group, 2019.

[52] M. Pérez-Ayúcar, M. Ashman, M. Almeida, M. Costa, Gregg, Science data volume management for the rosetta spacecraft, AIAA J. (2016). 
2021-05-21

\title{
Icarus: In-situ monitoring of the surface degradation on a near-sun asteroid
}

\author{
Lehtinen, Tuomas
}

Elsevier

Lehtinen T, Granvik M, Bellome A, Sanchez J-P. (2021) Icarus: In-situ monitoring of the surface degradation on a near-sun asteroid. Acta Astronautica, Volume 186, September 2021, pp. 98-108 https://doi.org/10.1016/j.actaastro.2021.05.028

Downloaded from Cranfield Library Services E-Repository 